\title{
Primary orbital hydatid cyst: computed tomography and magnetic resonance imaging findings
}

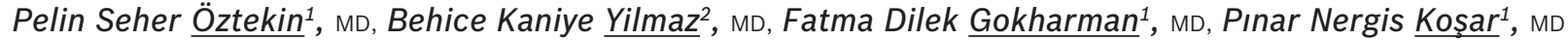

\begin{abstract}
Orbital hydatid cyst is a rare form of hydatidosis, comprising less than $1 \%$ of all hydatid cysts reported. The first choice of treatment for orbital hydatid cyst is surgery. Preoperative diagnosis is important, so as to avoid rupture of the cyst and prevent the spread of the parasitic disease. Herein, we present the computed tomography and magnetic resonance imaging findings of a case of primary orbital hydatid cyst.
\end{abstract}

Keywords: echinococcus granulosus, hydatid cyst, orbita

\section{INTRODUCTION}

Hydatid cyst is a parasitic disease caused by Echinococcus granulosus (E. granulosus). ${ }^{(1-3)}$ The disease is more common in the rural areas of South America, East and North Africa, and the Middle East, mainly in the Mediterranean countries, and is less common in developed countries. ${ }^{(4)}$ Hydatid cysts with orbital involvement are rare, comprising less than $1 \%$ of all systemic involvements of hydatid cysts. ${ }^{(1)}$

\section{CASE REPORT}

A 57-year-old man was admitted to the Neurosurgery Department with complaints of gradual loss of vision and swelling in his right eye that had lasted for two years. No additional pathologies were detected in his neurologic and systemic examinations. Routine blood tests and erythrocyte sedimentation rate were normal. The patient underwent paranasal-orbital computed tomography (CT) and magnetic resonance (MR) imaging, with a pre-diagnosis of a mass lesion in the right eye. A well-demarcated, cystic mass lesion measuring $1.5 \mathrm{~cm}$ and showing peripheral opacification was detected in the intraconal region (Figs. 1 \& 2). The lesion was adjacent to the lateral and medial rectus muscles, and extended to the antrum of the optic canal, exerting pressure on the optic nerve and resulting in displacement of the optic nerve (Fig. 3). No additional pathologies were detected on thorax CT or abdominal ultrasonography. Histopathological diagnosis of the mass, which was completely surgically excised, confirmed it to be a hydatid cyst (Fig. 4).

\section{DISCUSSION}

Hydatid cysts are most commonly located in the liver $(60 \%-$ $70 \%$ ) and the lungs $(20 \%){ }^{(3,4)}$ The orbita is a rare location for echinococcal infestation and makes up less than $1 \%$ of all cystic involvements. ${ }^{(5)}$ Hydatidosis of the orbit is more common among young adults and children. ${ }^{(6)}$ Other systemic involvements are rare in cases of orbital hydatid cyst. Orbital involvement is usually unilocular, without right or left dominancy. ${ }^{(2,3)}$ The cysts are usually located in the retrobulbar region, and may be
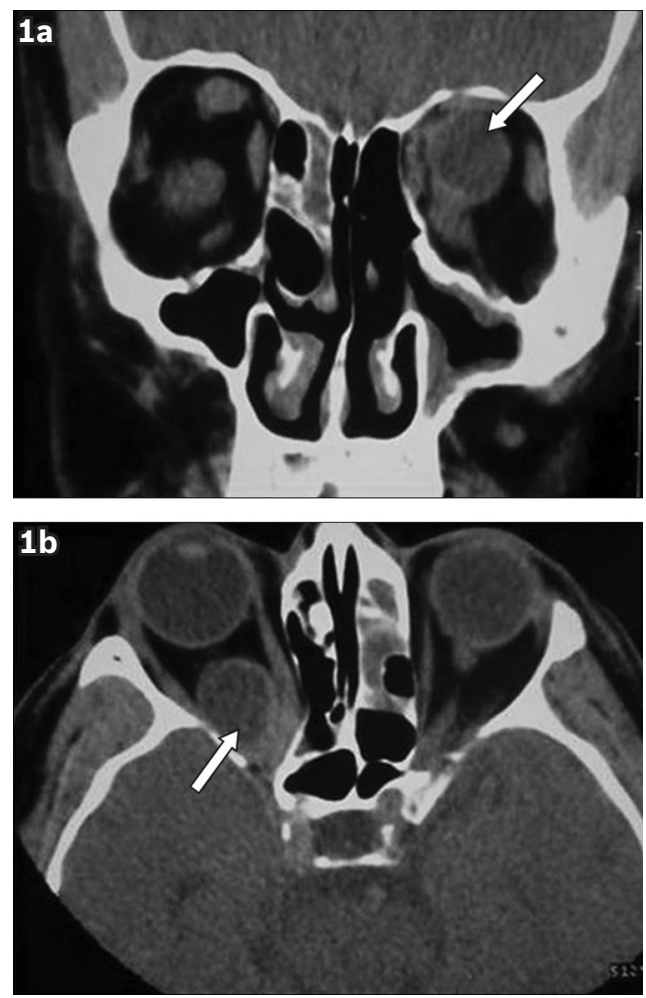

Fig. 1 (a) Coronal and (b) axial CT images show the well-demarcated, intraconal cystic lesion (arrows) localised in the posterosuperior region of the right bulbus oculi.

extraconal or, as in our case, intraconal. The superior quadrant of the visual field is usually affected in cases of extraconal involvement. ${ }^{(2)}$

Unilateral proptosis is the most common clinical finding in intraorbital hydatid cyst cases. ${ }^{(2,3)}$ Other findings include loss of vision, orbital pain, chemosis, palpebral oedema, and orbital cellulitis. Painless and slow-growing proptosis is a highly suspicious indicator of orbital hydatid cyst in endemic regions.

On CT, the orbital hydatid cyst is typically seen as a unilocular, non-enhancing homogeneous cyst with low density, similar to visualisation of the vitreous body. On MR imaging, the cyst shows isointense signalling on T1- and T2-weighted

${ }^{1}$ Department of Radiology, Ankara Training and Research Hospital, Ulucanlar, ${ }^{2}$ Department of Radiology, Hitit University Corum Training and Research Hospital, Merkez, Corum, Turkey

Correspondence: Dr Behice Kaniye Yilmaz, Registrar, Department of Radiology, Hitit University Corum Training and Research Hospital, Merkez, Corum 19100, Turkey. behiceyilmaz@gmail.com 



Fig. 2 Postcontrast (a) sagittal and (b) axial T1-W MR images demonstrate peripheral contrast enhancement in the cystic mass (arrows).


Fig. 3 Coronal (a) T1-W and (b) T2-W MR images show the mass effect of the cystic lesion (arrows) on the optic nerve.



Fig. 4 Photomicrograph shows the cuticle material and daughter cyst of the mass lesion (Haematoxylin \& eosin, $40 \times 10$ ).

images. In both CT and MR imaging, peripheral rim contrast is seen after the injection of a contrast medium. ${ }^{(1,6)}$ Coronal
CT is particularly beneficial in evaluating the accompanying bony pathologies, as they may erode different orbital cavity walls, depending on the anatomic localisations of these bony pathologies. ${ }^{(6)} \mathrm{MR}$ imaging is superior to $\mathrm{CT}$ with regard to the evaluation of the inner structure of the cyst and determining its association with neighbouring soft tissues. ${ }^{(3)}$ However, it is difficult to distinguish hydatid cysts from other mass lesions of the orbita via radiological imaging methods. Therefore, other cystic mass lesions like abscesses, mucocele, intraorbital haematomas, lacrimal tumours or cysts, and lymphangiomas must be considered in the differential diagnosis. . $^{(2,3,7}$

Surgery is the first treatment choice for orbital hydatid cyst, and a preoperative diagnosis is important in order to avoid rupture of the cyst and spread of the parasitic disease. ${ }^{(3,4)}$ In the differential diagnosis of orbital cysts in endemic areas, hydatid cysts should be one of the first diagnoses to be suspected. Although serological tests are used for diagnosis of hydatid cyst, serological tests are usually negative in cases of orbital involvement. Therefore, radiological imaging methods are valuable in the diagnosis of orbital hydatid cysts. ${ }^{(2,3)}$ 


\section{REFERENCES}

1. Turgut AT, Turgut M, Koşar U. Hydatidosis of the orbit in Turkey: results from review of the literature 1963-2001. Int Ophthalmol 2004; 25:193 200.

2. Torun F, Tuna H, Bozkurt M, Deda H. Orbital Hydatic Cyst: Case Report. Türk Nöroşirürji Dergisi 2004; 14:184-7.

3. Gökçek C, Gökçek A, Akif Bayar M, Tanrikulu S, Buharali Z. Orbital hydatid cyst: CT and MRI. Neuroradiology 1997; 39:512-5.
4. Ergün R, Okten Al, Yüksel M, et al. Orbital hydatid cysts: report of four cases. Neurosurg Rev 1997; 20:33-7.

5. Ciurea AV, Giuseppe G, Machinis TG, Coman TC, Fountas KN. Orbital hydatid cyst in childhood: a report of two cases. South Med J 2006; 99:620-4.

6. Morales AG, Croxatto JO, Crovetto L, Ebner R. Hydatid cyst of the orbit. A review of 35 cases. Ophthalmology 1988; 95:1027-32.

7. Fink AM, Newman DE, Stringer DA. Pediatric case of the day. Orbital hydatid cyst. Radiographics 1995; 15:731-4. 